\title{
Trichocyst Development During the Fission Cycle of Paramecium
}

\author{
By G. G. SELMAN AND A. JURAND \\ Institute of Animal Genetics, West Mains Road, Edinburgh, EH9 $3 \mathrm{JN}$
}

(Accepted for publication I3 November 1969)

\begin{abstract}
SUMMAR Y
In Paramecium aurelia, pretrichocysts originate in the endoplasm as undifferentiated vesicles. They develop in the endoplasm and then migrate to sites below the pellicle to become juvenile trichocysts. The maturation process takes place below the pellicle and involves a physical expansion and change in staining affinity of the body material of the trichocyst. Cold fixation usually caused mature trichocysts to be extruded, but the use of osmium tetroxide fixative at $37^{\circ}$ allowed them to be fixed without extrusion. Juvenile trichocysts cannot be extruded. In $P$. bursaria and $P$. aurelia after osmic fixation the body of the mature trichocyst, unlike the juvenile trichocyst, had no staining affinity for either potassium permanganate or toluidine blue. During fission in $P$. aurelia, mature trichocysts were present only in small numbers at the poles of the protozoa remote from the furrow. Juvenile trichocysts mature in the first hour of the interfission period.
\end{abstract}

\section{INTRODUCTION}

Ultrastructural observations on mature trichocysts of Paramecium were made by Sedar \& Porter (1955) with Paramecium multimicronucleatum and Stewart \& Muir (1963) with $P$. aurelia. Mature trichocysts were shown by Yusa (1963, I965) to be derived from juvenile trichocysts which developed in the endoplasm as pretrichocysts and which were themselves derived from undifferentiated endoplasmic vesicles. The terms pretrichocyst, juvenile trichocyst and mature trichochyst, used in this paper, were used by Yusa (1963, 1965). The developmental stages were illustrated in the electron micrographs of Yusa (I963) for P. caudatum, of Ehret \& De Haller (I963) for P. bursaria, and of Yusa (1965) for Frontonia vesiculosa. Frontonia has a close phylogenetic kinship to Paramecium according to Corliss (196I). All the pretrichocyst stages and juvenile trichocysts possess a distinctive crystalline body material which increases in volume as the pretrichocyst grows and develops the shape and structure characteristic of a juvenile trichocyst, so that the developmental sequence has probably been interpreted correctly from the electron micrographs. Moreover, most of the observations of Yusa (1963, I965) were made on the regeneration of trichocysts at recorded times after the mature trichocysts had all been discharged by the application of an electric current, so that observations on the development of a new set of mature trichocysts were made against a time scale which was initiated by the experimenter. For this reason, however, these observations of Yusa (1963, 1965) cannot be related to the normal fission cycle. Ehret \& De Haller (1963) made their observations during the normal fission cycle of $P$. bursaria and showed that pretrichocysts increased in 
number during the interfission interval. Although there is good agreement between these three accounts of the pretrichocyst stages, certain difficulties remain unresolved. For instance, Ehret \& De Haller (1963) depicted trichocysts with dense body material as mature trichocysts of $P$. bursaria, whereas Yusa $(1963,1965)$ showed that juvenile trichocysts attained maturity by a process in which the dense crystalline body of the juvenile trichocyst situated below the pellicle was apparently replaced by body material which was of low electron density and in which no crystalline periodicities were detected.

The present observations are mostly of the development and maturation of trichocysts in Paramecium aurelia, although some observations have also been made on $P$. bursaria. They confirm that in $P$. aurelia the pretrichocyst stages and juvenile trichocysts resemble those of $P$. bursaria and $P$. caudatum, and new results have been obtained concerning the behaviour of trichocysts during the normal fission cycle. Certain staining properties of juvenile trichocysts and difficulties involved in the fixation of mature trichocysts have been investigated.

\section{METHODS}

Cultures of Paramecium aurelia were used, mainly of stock 6o syngen I, but occasionally of other stocks. $P$. bursaria stock 93 syngen 2 was also used. Mass cultures were grown in an aqueous infusion of baked lettuce inoculated with Klebsiella (Aerobacter) aerogenes (Sonneborn, 1950). The protozoa were first filtered through a cotton plug and then concentrated into an aqueous pellet by low-speed centrifugation. The supernatant medium was decanted and the fixative added to the centrifuge tube, which was shaken to disperse the protozoa. A modified form of the fixative of Palade (1952) was prepared according to the formula quoted by Jurand \& Selman (1969). To preserve mature trichocysts the fixative was used at about $37^{\circ}$ instead of in the cold as normally recommended for electron microscopy. The penetration rate of the fixative is greater at the higher temperature. For comparative purposes fixations were also made by a modification of the method of Afzelius (I962), using a $30 \%$ (w/v) solution of osmium tetroxide in carbon tetrachloride. This method is fully described by Jurand \& Preer (1969) and has been proved suitable for the preservation of endosymbionts. Paramecia were fixed for $30 \mathrm{~min}$; in the case of warm fixations this period allowed the tube containing the protozoa to cool to room temperature. Dehydration was with graded ethanol + water mixtures and three changes of $100 \%$ ethanol (ro min. each). In bulk preparations the organisms were centrifuged down and the supernatant decanted at each stage before fresh ethanol was added. Finally, the protozoa were passed through a $50+50$ mixture I,2-epoxypropane + Araldite and embedded in Araldite by using a rotary shaker (Jurand \& Ireland, 1965). For light microscopy, Araldite sections were cut at I $\mu \mathrm{m}$. and stained with a $0.5 \%$ aqueous solution of toluidine blue made alkaline by adding I \% borax. Alternatively the staining procedure of Huber, Parker \& Odland (I968) was used. Ultrathin sections were cut on a Porter-Blum Sorvall ultramicrotome, mounted on collodion-carbon-coated grids and stained routinely for $20 \mathrm{~min}$. with a I $\%$ potassium permanganate solution containing $2.5 \%$ uranyl acetate. The sections were examined with an AEI EM 6 electron microscope.

Individual organisms were grown in medium containing bacteria using depression slides and an incubator at $24^{\circ}$. In this case individual paramecia were fixed, transferred 
through ethanol + water mixtures by using micropipettes, and embedded in separate capsules for subsequent examination in sections.

\section{OBSER VATIONS}

The ultrastructure of pretrichocysts and juvenile trichocysts in Paramecium aurelia may be observed after warm or cold fixation. Pretrichocyst stages are found in the endoplasm in positions which seem to bear no relationship to the trichocyst sites below the pellicle. Pretrichocysts are first recognizable by electron microscopy as irregularly rounded vesicles (P1. I, fig. I) containing amorphous material but with a dense oval core of crystalline material (Pl. I, fig. 4) with periodicities between 8 and $15 \mathrm{~nm}$. in different sections. The observed periodicity may depend upon the angle between the pretrichocyst and the plane of the section. At later pretrichocyst stages (Pl. I, fig. 2, 3) the vesicle and its dense crystalline core are larger. The core becomes elongated and develops a tip (Pl. I, fig. 3). Then a sheath or cap forms round the tip and migration to the pellicle takes place (Pl. I, fig. 7). Yusa (1963, 1965) did not define the exact developmental stage beyond which a pretrichocyst is termed a juvenile trichocyst, but in the present paper the term juvenile trichocyst is applied after migration to the pellicle has occurred. In the juvenile trichocyst there is no discontinuity between the tip and the body, and both share the same crystalline structure (Pl. I, fig. 6). In the electron micrographs sets of parallel dark lines run perpendicularly to the axis of the juvenile trichocyst at intervals of 7 to $8 \mathrm{~nm}$. and further sets of dark lines with intervals of $\mathrm{I} 2 \mathrm{~nm}$. intersect the first set at $65^{\circ}$ (PI. I, fig. 6). Dense peripheral granules, about $13 \mathrm{~nm}$. in diameter, occur outside the body material of juvenile trichocysts and just within their limiting membrane (Pl. I, fig. 5).

In experimental batches of material juvenile trichocysts and pretrichocysts were observed by electron microscopy after osmic fixation but without additional heavymetal stain. Under these conditions no crystalline structure was observed in pretrichocysts or in the body or tip of juvenile trichocysts; only a slight darkening was observed round their edges, although in the same sections cytoplasmic membranes and the membranes of mitochondria appeared dense and sharply defined. The dense appearance of the juvenile trichocyst body in material prepared by the normal procedures is not therefore due to combination with osmium tetroxide as was assumed in Selman \& Jurand (I968). When osmic fixation was followed by the use of potassium permanganate but without uranyl acetate, the pretrichocysts and juvenile trichocysts showed dense crystalline structure. When osmic fixation was followed by staining with uranyl acetate alone, the density of staining was less than with potassium permanganate and crystalline structure was not observed. Staining with lead citrate alone (Reynolds, I963) gave a uniform dense stain which was not as intense as after potassium permanganate when the staining times were similar. It may be concluded that whenever juvenile trichocysts appear dense by electron microscopy it is because they combine with the heavy-metal stains which have been used after osmic fixation. The use of these stains has also enabled their crystalline ultrastructure to be observed.

The fixation and embedding of a concentrated mass culture of Paramecium aurelia allows a batch of several hundred protozoa in all stages of the fission cycle to be examined rapidly by light or electron microscopy after serial sectioning. When warm fixation was used, the mature trichocysts were not found extruded into the medium 
between the protozoa but they were observed in their normal sites below the pellicle, their sheathed tips alternating with the basal granules or pairs of basal granules down each kinety (see also Ehret \& Powers, 1959). The body of each mature trichocyst showed no affinity for heavy-metal stains and appeared white and without ultrastructure in electron micrographs ( $\mathrm{Pl}$. 2, fig. 8, 9). The tip of the mature trichocyst appeared dense and showed crystalline structure with regular cross-striations ( $\mathrm{Pl}$. 2, fig. 9).

When cold fixation was used, the shafts of extruded trichocysts were found in the medium between the protozoa (Pl. 2, fig. I I) and no mature trichocysts, but only immature juvenile trichocysts, were seen below the pellicle. Such juvenile trichocysts are probably incapable of extrusion. In some cases, cold fixation caused mature trichocysts to be extruded backwards into the cytoplasm. In extrusion the trichocyst body becomes transformed into an elongated shaft and then shows transverse striations (see Jakus \& Hall, I946) with a main periodicity of about $50 \mathrm{~nm}$. (Pl. 2, fig. I2) and some fine longitudinal fibrillar ultrastructure with a fibril diameter of about $3 \mathrm{~nm}$. In some cases a trichocyst was observed only partially extruded. In these cases the body was curved and thinner than a mature trichocyst but thicker than a fully extruded shaft.

When mass cultures of Paramecium were sectioned at $\mathrm{I} \mu \mathrm{m}$. and stained with toluidine blue for light microscopy it was immediately obvious that mature trichocysts remained unstained and juvenile trichocysts were deeply stained (Pl. 2, fig. 10). Therefore when a juvenile trichocyst becomes a mature trichocyst the result of the change which takes place in its body material can be seen by light microscopy as easily as by electron microscopy (Pl. 2, fig. I0, compare with Pl. 3, fig. I3). No stages of intermediate staining density were observed, however, between the juvenile and the mature trichocyst either by light or electron microscopy. When the staining method of Huber et al. (I968) was used, the juvenile trichocysts stained with both basic fuchsin and methylene blue and appeared dark purple against the pink background of the cytoplasm, while mature trichocysts did not stain. When unstained sections, I $\mu \mathrm{m}$. thick, were observed by positive phase-contrast microscopy, the juvenile trichocysts appeared optically denser than mature trichocysts.

Measurements of the body length and the widest cross-sectional diameter of juvenile and mature trichocysts were made on electron micrographs of trichocysts seen in longitudinal section at their sites below the pellicle. Means and standard deviations were calculated. Juvenile trichocysts had a diameter of $0.70 \pm 0.14 \mu \mathrm{m}$. and body length of $2 \cdot 70 \pm 0.53 \mu \mathrm{m}$., whereas mature trichocysts had a diameter of $1 \cdot 27 \pm 0.26 \mu \mathrm{m}$. and body length of $5.02 \pm 0.72 \mu \mathrm{m}$. Therefore the mature trichocyst has a body which is about $84 \%$ greater in its mean linear dimensions than the juvenile trichocyst and has a mean volume six times greater than the mean volume of the juvenile trichocyst. Moreover, the individual values for the corresponding dimension in mature and juvenile trichocysts form two groups which do not overlap. Measurements and observations of staining affinity thus support each other and suggest that the change from a juvenile to a mature trichocyst is rapid and involves both an expansion and a change in the chemical properties of the body material.

Mass cultures of Paramecium bursaria were examined after warm fixation by both light and electron microscopy. As with $P$. aurelia, mature trichocysts were fixed without extrusion and these had bodies without affinity for potassium permanganate or 
toluidine blue. The juvenile trichocysts densely stained both with potassium permanganate and with toluidine blue (Pl. 2, fig. IO). The only difference was the considerable proportion of partially extruded mature trichocysts which were found in $P$. bursaria after warm fixation. In $P$. aurelia these may occur after cold but not after warm fixation. The difference may indicate that trichocysts of $P$. bursaria are more easily extruded.

A careful examination of sectioned material from mass cultures of Paramecium aurelia after warm fixation showed that, except for organisms in fission, the proportion of juvenile to mature trichocysts was usually small but varied with the individual protozoon. Whenever the fission furrow could be seen, the paramecia consistently showed a complete lack of mature trichocysts over about nine-tenths of their surface (Pl. 3, fig. I5). No mature trichocysts were seen near the furrow or in the middle part of the body of these paramecia, although many juvenile trichocysts were observed below these regions of the pellicle. A few mature trichocysts were observed, however, at the extreme ends of paramecia in fission (Pl. 3, fig. I4, I5). The dividing protozoa were not surrounded by extruded trichocysts, so that the lack of mature trichocysts is probably not due to a higher sensitivity of such protozoa to extrusion in the presence of fixative. The majority of mature trichocysts seemed to have been lost immediately before fission. It also appears that the juvenile trichocysts present in the fission animal must attain maturity soon after fission since the mass cultures showed that, except for dividing organisms, most paramecia had a majority of mature trichocysts. Observations were accordingly made on individually fixed organisms where an estimate could be made of the stage with respect to the fission cycle at the time of fixation.

In an experiment at $25^{\circ}$ the average fission period was $8 \mathrm{hr} 40 \mathrm{~min}$, , but individual fission intervals varied over a range of $2 \mathrm{hr}$. In paramecia fixed I $\mathrm{hr}$ after the previous fission, $9 \mathrm{I} \%$ of the trichocysts below the pellicle were mature and the remainder were juvenile. It was concluded, therefore, that the majority of the juvenile trichocysts matured immediately after fission. In paramecia fixed at an estimated time of $\mathrm{I} \mathrm{hr}$ before fission, $98 \%$ of the trichocysts below the pellicle were mature. It was concluded that mature trichocysts are lost immediately before fission takes place. The mature trichocysts may discharge themselves before fission or they may degenerate.

\section{DISCUSSION}

The present observations show that mature trichocysts of Paramecium aurelia are almost completely absent from paramecia in fission, being normally discharged immediately before fission; the discharge itself has not been observed. Alternatively, the mature trichocysts might degenerate before fission, but this is less probable since degenerating trichocysts have been observed in only a few cases and then in the endoplasm rather than below the pellicle. Juvenile trichocysts are present below the pellicle during fission and subsequently attain maturity soon after fission. These conclusions were unexpected since it had been assumed previously that mature trichocysts are structurally stable components of the pellicle and are distributed between the two daughters at fission, while an equal number of new trichocysts develop from pretrichocysts during each fission cycle. The work of Ehret, Savage \& Alblinger (I964) seems to support the conservation of mature trichocysts through cell fission, since they showed that when trichocysts of $P$. bursaria were labelled with tritiated leucine they retained the label without dilution for at least three fission generations thereafter. 
The present work suggests that this may be true only for trichocysts located at the two poles of the paramecia remote from the fission furrow.

The mature trichocysts of Paramecium bursaria and $P$. aurelia have bodies composed of material in which no regular structure has been observed. This apparent lack of ultrastructure may be due to the lack of an appropriate stain. It is most unlikely that the mature trichocyst has an amorphous body since its rapid extrusion involves another change of state, after which regular periodicities are observed once more, although the ultrastructure of extruded shafts is quite different from that of juvenile trichochysts. Ehret \& De Haller (1963) did not record the maturation of trichocysts in $P$. bursaria and their electron micrographs show only juvenile trichocysts. Ehret $\&$ Powers (1957, 1959) published electron micrographs in which mature trichocysts may be recognized. The origin and development of trichocysts in $P$. aurelia is similar to their epigenesis in $P$. caudatum (Yusa, 1963), P. bursaria (Ehret \& De Haller, I963) and Frontonia vesiculosa (Yusa, 1965). Ultrastructural studies have indicated that trichocysts develop from endoplasmic vesicles, although these vesicles cannot be identified as pretrichocysts unless they possess at least some of the crystalline material of which the body and tip of juvenile trichocysts is composed. It is not known how these vesicles originate and there is no evidence from any work with Paramecium species that they are formed in association with any type of organelle. It is therefore difficult to imagine in what sense trichocysts can continue to be regarded as ultrastructural homologues of cilia (e.g. see Ehret, 1967), although Lwoff (1950), on the basis of observations with the light microscope, considered that the trichocysts of certain parasitic ciliates are derived from kinetosomes.

The body material of trichocysts appears to exist in different physical states, one of which occurs in pretrichocysts and juvenile trichocysts, another in mature trichocysts and a third in extruded trichocysts. Changes between these states appear to take place quickly and so far as is known irreversibly and involve changes in dimensions and staining affinity. Regular periodicities have been directly observed in pretrichocysts, juvenile trichocysts and extruded trichocysts, but their presence in the body material of mature trichocysts seems likely but has not yet been directly observed.

We wish to thank Professor G. H. Beale, F.R.S., for his interest and for his comments on a draft of this paper.

\section{REFERENCES}

Afzelius, B. A. (1962). Chemical fixatives for electron microscopy. In The Interpretation of Ultrastructure. Ed. by R. J. C. Harris, p. I. New York: Academic Press.

Corliss, J. O. (196I). The Ciliated Protozoa. New York: Academic Press.

EHRET, C. F. (1967). Paratene theory of the shapes of cells. Journal of Theoretical Biology 15, 263.

EhreT, C. F. \& DE Haller, G. (I963). Origin, development and maturation of organelles and organelle systems of the cell surface in Paramecium. Journal of Ultrastructure Research 6 (Suppl.), 2.

Ehret, C. F. \& Powers, E. L. (I957). The organization of gullet organelles in Paramecium bursaria. Journal of Protozoology 4, 55.

EHret, C. F. \& Powers, E. L. (1959). The cell surface of Paramecium. International Review of Cytology $8,97$.

Ehret, C., Savage, N. \& Alblinger, J. (I964). Patterns of segregation of structural elements during cell division. Zeitschrift für Zellforschung und Mikroskopische Anatomie 64, I29.

Huber, J. D., Parker, F. \& Odland, G. F. (1968). A basic fuchsin and alkalinized methylene blue rapid stain for epoxy-embedded tissue. Stain Technology 43,83 . 
JAKUS, M. A. \& HALL, C. E. (1946). Electron microscope observations of the trichocysts and cilia of Paramecium. Biological Bulletin. Marine Biological Laboratory, Woods Hole, Mass. 9x, I4I.

Jurand, A. \& IRELAND, M. J. (1965). A slow rotary shaker for embedding in viscous media. Stain Technology 40, 233.

Jurand, A. \& Preer, L. B. (I969). Ultrastructure of flagellated lambda symbionts in Paramecium aurelia. Journal of General Microbiology 54, 359.

Jurand, A. \& Selman, G. G. (1969). The Anatomy of Paramecium aurelia. London: Macmillan.

Lwoff, A. (1950). Problems of Morphogenesis in Ciliates. New York: John Wiley and Sons.

PALADE, G. E. (1952). A study of fixation for electron microscopy. Journal of Experimental Medicine $95,285$.

Reynolds, E. S. (1963). The use of lead citrate at high pH as an electron-opaque stain in electron microscopy. Journal of Cell Biology $\mathbf{1 7}, 208$.

Sedar, A. W. \& Porter, K. R. (I955). The fine structure of cortical components of Paramecium multimicronucleatum. Journal of Biophysical and Biochemical Cytology $\mathbf{r}, 583$.

Selman, G. G. \& JuRAnd, A. (I968). The formation of cell organelles during the fission cycle of Paramecium aurelia. Journal of Protozoology 5 (Suppl.), 34.

Sonneborn, T. M. (1950). Methods in the general biology and genetics of $P$. aurelia. Journal of Experimental Zoology $113,87$.

Stewart, J. M. \& Muir, A. R. (1963). The fine structure of the cortical layers in Paramecium aurelia. Quarterly Journal of Microscopical Science 104, 129.

YUSA, A. (1963). An electron microscope study on regeneration of trichocysts in Paramecium caudatum. Journal of Protozoology ro, 253.

YusA, A. (1965). Fine structure of developing and mature trichocysts in Frontonia vesiculosa. Journal of Protozoology 12, $5 \mathrm{I}$.

\section{EXPLANATION OF PLATES}

All figures are of Paramecium aurelia except for Fig. Io which is of $P$. bursaria. Fig. I to 9 and II to I 4 are electron micrographs. Fig. 10 and 15 are light micrographs. All scales correspond to $1 \mu \mathrm{m}$. unless otherwise indicated.

\section{Plate I}

Fig. I. An endoplasmic pretrichocyst at an early stage bounded by a membrane and containing a dense central core of crystalline material. $\times 28,000$.

Fig. 2. A pretrichocyst at a slightly later stage than in Fig. I. The crystalline core is elongated. $\times 28,000$.

Fig. 3. A pretrichocyst from the endoplasm at a later stage than Fig. 2. The tip has developed in continuity with the body. There has been a decrease in the amount of finely dispersed material between the body and the membrane of the pretrichocyst. $\times 28,000$.

Fig. 4. A detail of the crystalline core of a pretrichocyst at an early stage as in Fig. I. The regular periodicities are shown. $\times 93,000$.

Fig. 5. A detail of a juvenile trichocyst similar to that shown in Fig. 7 to show the periodicities in the body material. Note also a single row of dense granules between the membrane and the dense body. $\times 93,000$.

Fig. 6. Part of a juvenile trichocyst to show the continuity between its tip and body and the similarity of the crystalline structure in the two regions. $\times 93,000$.

Fig. 7. A juvenile trichocyst in longitudinal section in its position below the pellicle. Round the tip a sheath has developed which was not present at the late pretrichocyst stage shown in Fig. 3 . $\times 28,000$.

\section{Plate 2}

Fig. 8. Mature trichocysts are shown in longitudinal section at their sites below the pellicle. The bodies appear unstained and electron-transparent, in contrast to the juvenile trichocyst in Fig. $7 . \times 7600$. Fig. 9. A detail from a mature trichocyst to show the region of the join between the dense crystalline tip and the electron-transparent body material. Contrast with the juvenile trichocyst in Fig. 6. $\times 85,000$.

Fig. Io. Mature $(m)$ and juvenile trichocysts $(j)$ are shown at the surface of $P$. bursaria after warm fixation with osmium tetroxide. Algal symbionts $(a)$ are present in the cytoplasm. Light micrograph stained with toluidine blue. $\times 1400$. 
Fig. II. In $P$. aurelia fixed with cold osmium tetroxide the shafts $(s)$ of discharged trichocysts are present in the medium at the cell surface. Only darkly stained juvenile trichocysts $(j)$ remain undischarged below the pellicle. Electron micrograph stained with potassium permanganate and uranyl acetate. $\times$ I I, 000.

Fig. 12. The shaft and tip of an extruded trichocyst of $P$. aurelia, outside the animal. Transverse striations occur in the shaft at intervals of $50 \mathrm{~nm} . \times 38,000$.

\section{Plate 3}

Fig. 13. Both mature $(m)$ and juvenile trichocysts $(j)$ are shown below the pellicle of $P$. aurelia. The trichocysts are seen in longitudinal and transverse section. The juvenile trichocysts appear smaller and darkly stained. $\times 4700$.

Fig. 14. The polar region of a paramecium in fission shows two mature trichocysts $(m)$ in the plane of the section and several juvenile trichocysts $(j)$ below the pellicle. $\times 6600$.

Fig. 15. The longitudinal section through a paramecium in fission shows the fission furrow and one polar region remote from the furrow. Only the polar region contains a group of mature trichocysts $(m)$, which appear white, while only darkly stained juvenile trichocysts are present below the pellicle over the remainder of the body surface. Warm fixation; stained with toluidine blue. $\times 1400$. 
Journal of General Microbiology, Vol. 60, No. 3
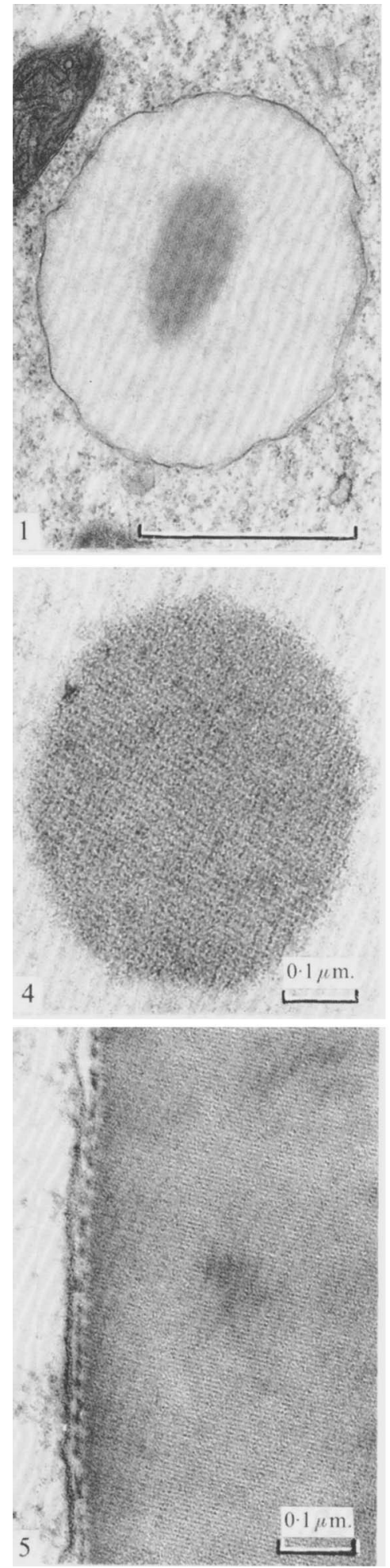
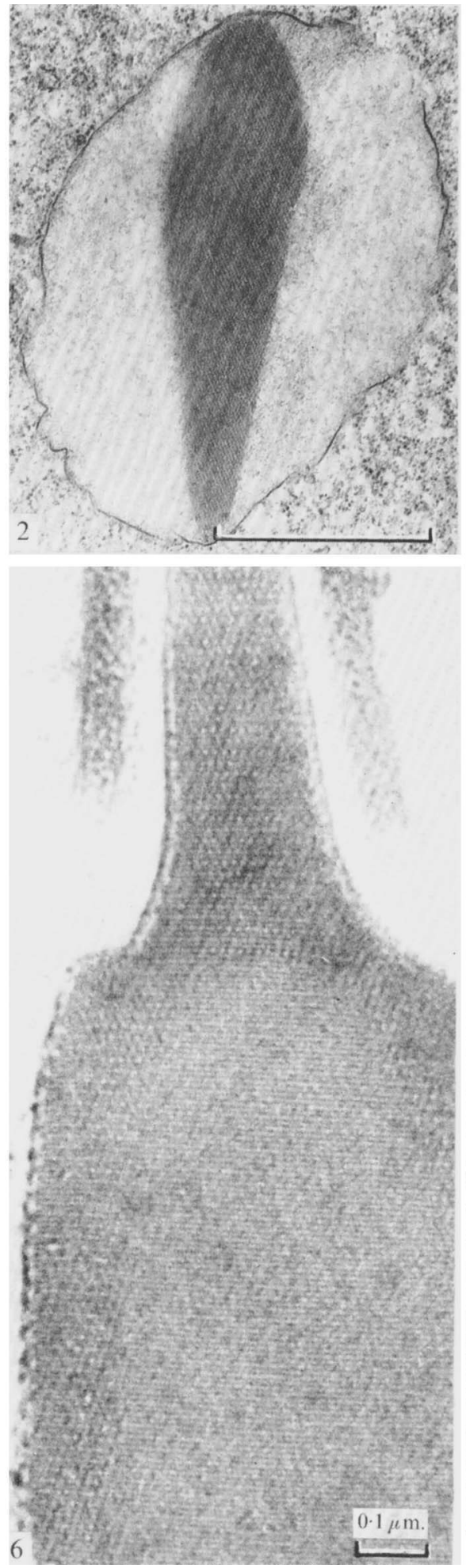

G. G. SELMAN ANI A. JURAND

Plate I
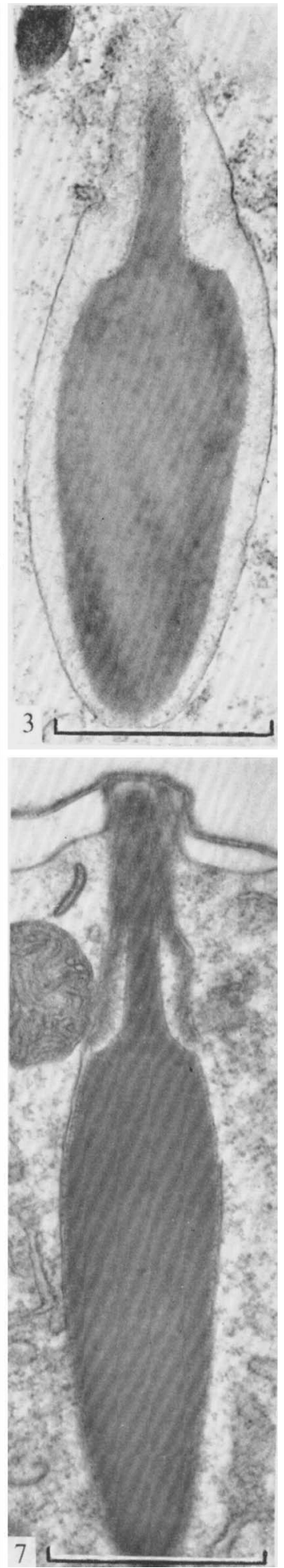

(Facing p. 372) 
Journal of General Microbiology, Vol. 6o, No. 3

Plate 2
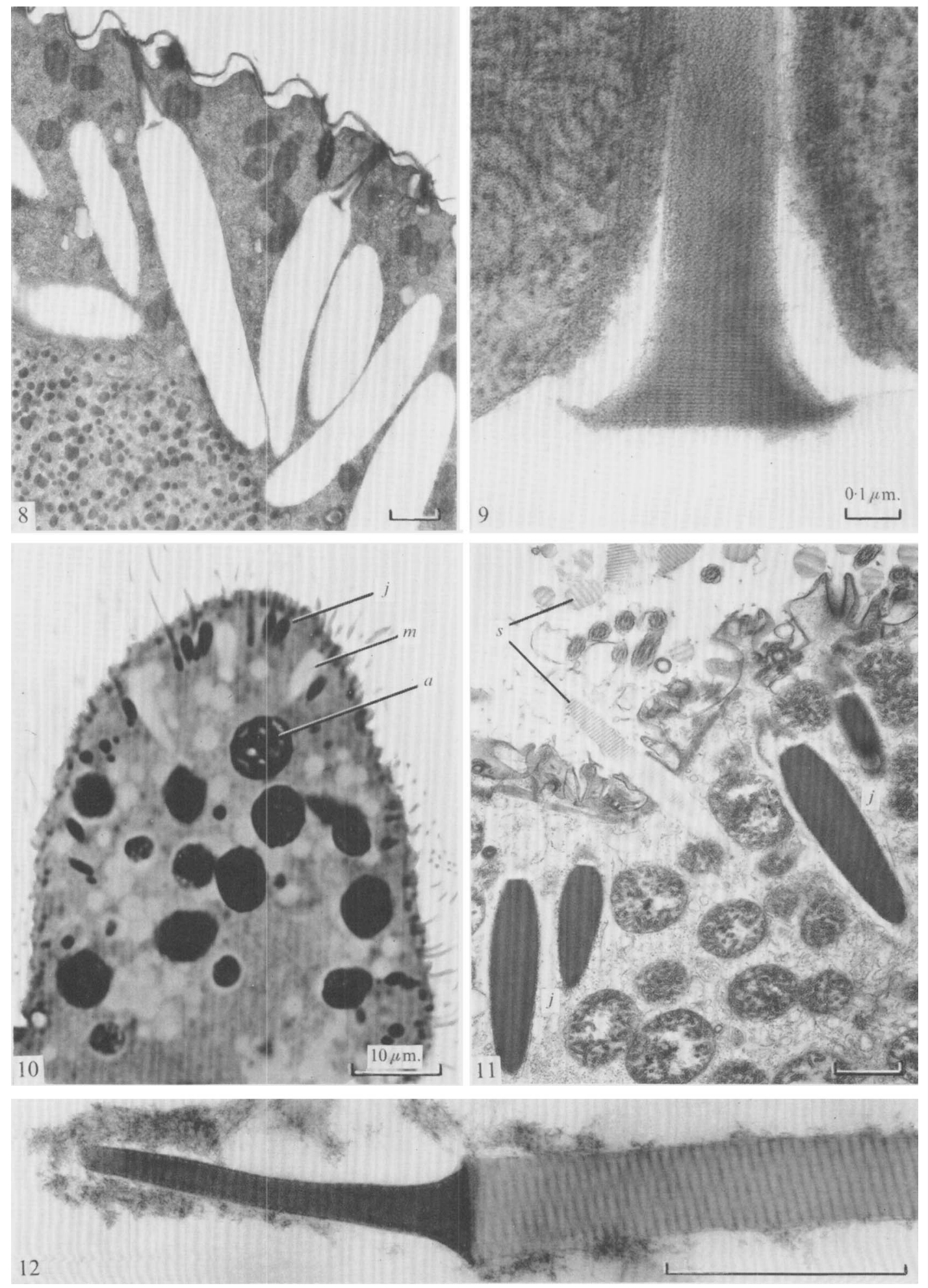

G. G. SELMAN AND A JURAND 
Journal of General Microbiology, Vol. 6o, No. 3

Plate 3
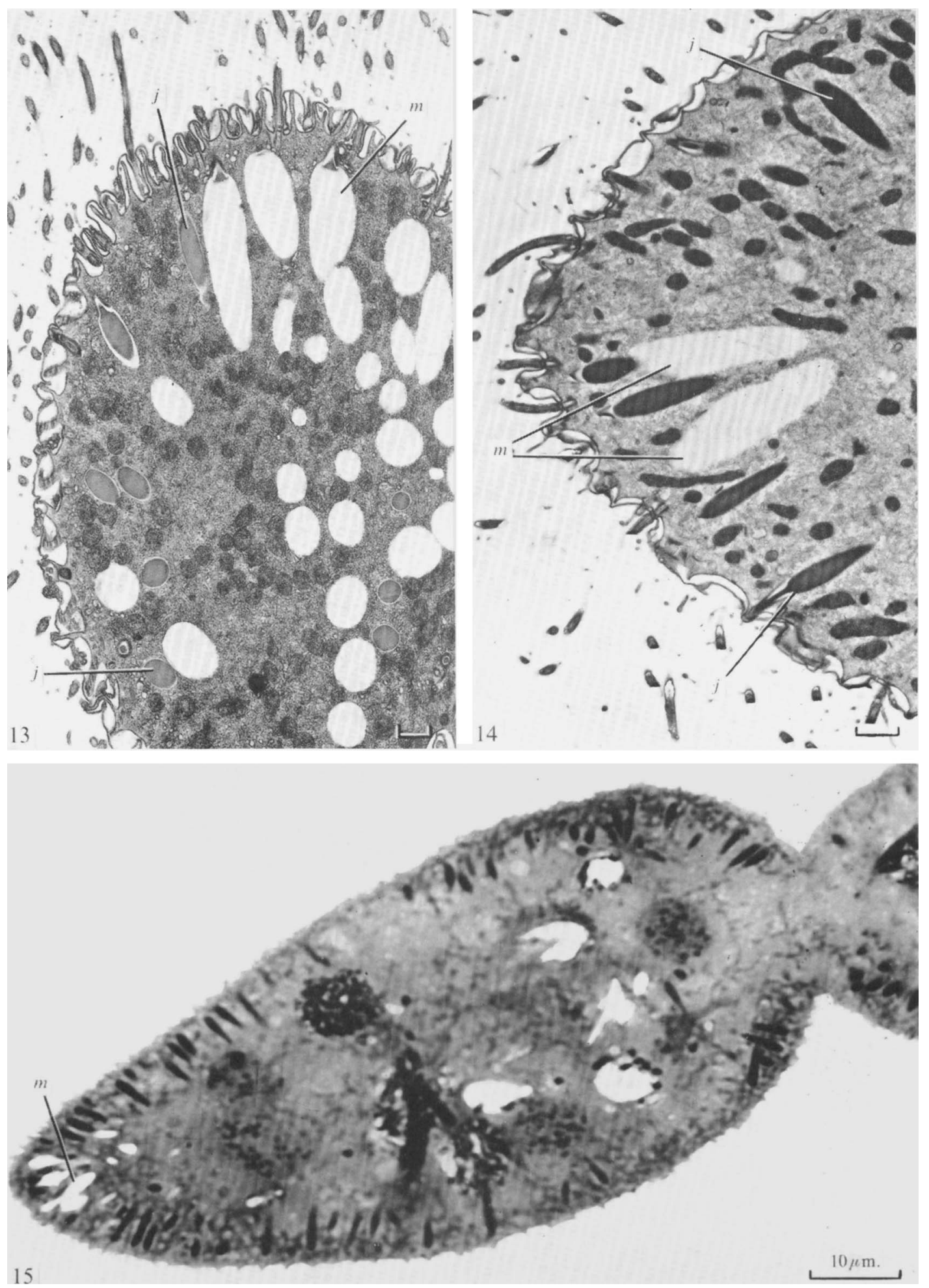

G. G. SELMAN AND A. JURAND 